\title{
The Exact Absorbing Conditions Method in the Analysis of Open Electrodynamic Structures: Circular and Coaxial Waveguides
}

\author{
Seil S. Sautbekov, ${ }^{1}$ Yuriy K. Sirenko, ${ }^{1,2}$ Lyudmyla G. Velychko, ${ }^{2}$ and Alexey A. Vertiy \\ ${ }^{1}$ L.N. Gumilyov Eurasian National University, 5, Kazhimukan Street, Astana 010008, Kazakhstan \\ ${ }^{2}$ A.Y. Usikov Institute of Radiophysics and Electronics, National Academy of Sciences of Ukraine, 12 Acad. Proskura Street, \\ Kharkiv 61085, Ukraine
}

Correspondence should be addressed to Yuriy K. Sirenko; yks@bk.ru

Received 28 November 2013; Accepted 25 March 2014; Published 23 April 2014

Academic Editor: Stefano Selleri

Copyright (C) 2014 Seil S. Sautbekov et al. This is an open access article distributed under the Creative Commons Attribution License, which permits unrestricted use, distribution, and reproduction in any medium, provided the original work is properly cited.

\begin{abstract}
The exact absorbing conditions (EAC) have been constructed and used for truncating an unbounded domain of computation in open initial boundary value problems, which describe space-time transformations of electromagnetic waves in axially symmetrical waveguides. The equivalence theorem is proved that gives grounds for rigorous theoretical justification of the EAC-method.
\end{abstract}

\section{Introduction}

Exact absorbing conditions (EAC) are used in computational electrodynamics of nonsine waves for truncating the domain of computation when replacing the original open initial boundary value problem by a modified problem formulated in a bounded domain [1-9]. In the present work, we construct and analyze the EAC as applied to the axially symmetrical waveguide structures illuminated by symmetrical pulsed TEand $\mathrm{TM}$-waves (or $\mathrm{TE}_{0}$ - and $\mathrm{TM}_{0}$-waves) and prove the equivalency of the original (open) and modified (closed) initial boundary value problems. The following results are presented: the EAC for virtual boundaries in a cross-section of regular circular and coaxial waveguides and the theorem about the one-valued solvability of the modified closed problem and its equivalency to the initial open problem giving grounds for rigorous theoretical justification of the EAC-method.

The efficient limitation of the computational space in open initial boundary value problems (i.e., the problems whose domain of analysis is infinite in one or more directions) is a vital issue in computational electrodynamics as well as in other physical disciplines dealing with mathematical simulation and numerical experiments. Most of the well-known and extensively used heuristic and approximate solutions to this problem are based on the so-called absorbing boundary conditions (ABC) [10-13] and perfectly matched layers (PML) [14-16]. The use of various modifications and improving techniques for respective methods yield good results in various specific physical situations. However, it appears that, for certain problems associated with the resonant wave scattering, the numerical implementation of these methods may cause unpredictable growth of the computational error for large observation times [17-19].

The method utilizing the exact absorbing conditions for the artificial boundaries that truncate an unbounded domain of computation $[1-9,20,21]$ is outnumbered by the classical approximate approaches. However, testing numerical experiments as well as a series of physical and applied results obtained with the help of this method (see, e.g., [3, 5, 8, 2130]) shows its evident potential, especially, for obtaining reliable numerical data on space-time and space-frequency electromagnetic field transformations in open waveguide, periodic, and compact resonators.

The essence of the method for open scalar problems, which are formulated in the part $\Omega$ of the space $R^{2}\left(R^{n}\right.$ is $n$ dimensional Euclidean space), is as follows. Assume that the excitation sources and inhomogeneities of the medium are located in a bounded region $\Omega_{\text {int }}$ of the unbounded analysis domain $\Omega$. The propagation velocity of the electromagnetic 
wave $U(g, t), g \in \Omega$, generated by these sources and obstacles is finite. Therefore, in time $t \leq T<\infty$, the signal $U(g, t)$ will not go outside the boundaries $\Pi_{T}$ of some bounded domain $\Omega_{\text {int }, T} \subset \Omega$ :

$$
\left.U(g, t)\right|_{g \in \Omega_{\mathrm{ext}, T}, t \in[0, T]}=0 .
$$

Here, $[0, T]$ is the closed interval $0 \leq t \leq T$ and $\Omega_{\text {ext, } T}$ is the complement of the domain $\Omega_{\mathrm{int}, T}$ with respect to $\Omega$; that is, $\Omega=\Omega_{\text {int }, T} \cup \Pi_{T} \cup \Omega_{\text {ext }, T}$.

Formula (1) gives us the well-known exact radiation condition for outgoing (from the domain $\Omega_{\text {int }}$ where all sources and obstacles are located) pulsed wave $U(g, t)$. The only but rather essential limitation of this simple condition is that, with growing $T$, the domain $\Omega_{\mathrm{int}, T}$ is expanding, and the boundary $\Pi_{T}$ is moving farther away from the domain $\Omega_{\text {int }}$. That is why the condition in the form of (1) is not used for truncating the computational space of open electrodynamic problems. In EAC-method, the condition (1) is transferred from the field-free points $g \in \Omega_{\text {ext, } T}$ onto some artificial boundary $\Gamma$ located in the region, where the intensity of spacetime field transformations can be arbitrary in magnitude and takes the form

$$
\left.D[U(g, t)]\right|_{g \in \Gamma}=0 ; \quad t \geq 0 .
$$

The electromagnetic wave $U(g, t)$ must be outgoing in this case as well, or, in other words, it is bound to intersect the boundary $\Gamma$ only in one direction, moving away from the sources and obstacles. Here, as above, $U(g, t)(g \in \Omega, t \geq$ $0)$ is a scalar or vector field function, while $D[U]$ is some integrodifferential operator on $\Gamma \times[0, \infty),[0, \infty)=\{t: 0 \leq$ $t<\infty\}$. Symbol " $x$ " denotes direct product of two sets.

The boundary $\Gamma$ divides the unbounded domain $\Omega$ into two domains $\Omega_{\text {int }}$ and $\Omega_{\text {ext }}$ such that $\Omega=\Omega_{\text {int }} \cup \Omega_{\text {ext }} \cup$ $\Gamma$. In the first one (bounded), we can formulate the initial boundary value problem with respect to the function $U(g, t)$ using the boundary condition in (2). We will further call this problem the modified problem as distinct from the original initial boundary value problem formulated in the unbounded domain $\Omega$ with the radiation condition (1) involved. In the domain $\Omega_{\text {int }}$, the desired function $U(g, t)$ can be determined by using standard finite-difference [31] or finite element [32] algorithms. For the domain $\Omega_{\text {ext }}$, EAC-method allows us to construct and use the so-called "transport operators" $Z_{q \in \Gamma \rightarrow g \in \Omega_{\text {ext }}}(t)[U][3,8,33,34]$

$$
U(g, t)=Z_{q \in \Gamma \rightarrow g \in \Omega_{\mathrm{ext}}}(t)[U(q, \tau)], \quad 0 \leq \tau \leq t,
$$

to calculate the function $U(g, t)$ at the points $g \in \Omega_{\text {ext }}$ from its values on the boundary $\Gamma$.

The analytical forms of the operators $D[U]$ and $Z[U]$ depend on the geometry of the domain $\Omega_{\text {ext }}$, and, evidently, on the problem dimensions and the coordinate system. However, in all cases, the derivation of these operators is based on the common sequence of transformations widely used in the theory of hyperbolic equations $[3,35]$ : (i) the isolation of the regular domain $\Omega_{\text {ext }}$ where the wave $U(g, t)$ propagates freely moving away from the domain $\Omega_{\text {int }}$ enveloping all sources and scattering objects, (ii) incomplete separation of variables in the original initial boundary value problem for the domain $\Omega_{\text {ext }}$ resulting in the problem for the one-dimensional KleinGordon equation with respect to the space-time amplitudes of the field $U(g, t)$, (iii) integral transformation (image $\leftrightarrow$ original function) of the problem for one-dimensional KleinGordon equation, (iv) solution of auxiliary boundary value problems for ordinary differential equations with respect to the images of amplitudes of the field $U(g, t)$, and (v) inverse integral transformation.

As a result, the nonlocal (in space and time) exact absorbing conditions on the artificial boundary $\Gamma$ are derived. In some cases, these nonlocal conditions can be reduced to the local conditions by replacing certain integral forms with the differential forms and defining an additional initial boundary value problem with respect to some auxiliary function of time and transverse coordinates $[2,3,8]$. The exact EAC (2) can be then included into a standard finite-difference algorithms or into algorithms of finite element method with the domain of calculation reduced down to $\Omega_{\text {int }}$. However, one can confidently assert that relevant computational schemes are stable and convergent only when the modified problem is uniquely-solvable and equivalent to the original problem [36]. Although the corresponding assertions have been formulated in some works on the subject (see, e.g., [1, 3, $8]$ ), they have been proved analytically just only in [9] for the initial boundary value problems describing $\mathrm{TE}_{0}$ - and $\mathrm{TM}_{0^{-}}$ pulsed wave scattering on compact open axially symmetrical structures. In Section 6, we replicate the proof scheme for the initial boundary value problem associated with compact discontinuities in circular and coaxial waveguides and thus demonstrate that there is reason to believe that it can be used for the other types of EAC as well.

\section{Formulation of the Model Problem}

The two-dimensional initial boundary value problem describing the transformation of pulsed symmetrical $(\partial / \partial \phi \equiv 0)$ $\mathrm{TE}_{0}-\left(E_{\rho}=E_{z}=H_{\phi} \equiv 0\right)$ and $\mathrm{TM}_{0}$-waves $\left(H_{\rho}=H_{z}=E_{\phi} \equiv\right.$ 0 ) in the open axially symmetrical waveguide units (Figure 1) is given $($ see $[3,8])$ by

$$
\begin{gathered}
{\left[-\varepsilon(g) \frac{\partial^{2}}{\partial t^{2}}-\sigma(g) \eta_{0} \frac{\partial}{\partial t}+\frac{\partial^{2}}{\partial z^{2}}+\frac{\partial}{\partial \rho}\left(\frac{1}{\rho} \frac{\partial}{\partial \rho} \rho\right)\right]} \\
\times U(g, t)=F(g, t) ; \quad t>0, g \in \Omega, \\
\left.U(g, t)\right|_{t=0}=\varphi(g),\left.\quad \frac{\partial}{\partial t} U(g, t)\right|_{t=0}=\psi(g) ; \\
g=\{\rho, z\} \in \bar{\Omega}, \\
\left.E_{t g}(p, t)\right|_{p=\{\rho, \phi, z\} \in \Sigma}=0, \quad U(0, z, t)=0 \\
\text { for }|z|<\infty ; t \geq 0 .
\end{gathered}
$$

Here, $\vec{E}=\left\{E_{\rho}, E_{\phi}, E_{z}\right\}$ and $\vec{H}=\left\{H_{\rho}, H_{\phi}, H_{z}\right\}$ are the electric and magnetic field vectors, $\{\rho, \phi, z\}$ are the cylindrical coordinates, $U(g, t)=E_{\phi}(g, t)$ for $\mathrm{TE}_{0}$-waves, and 


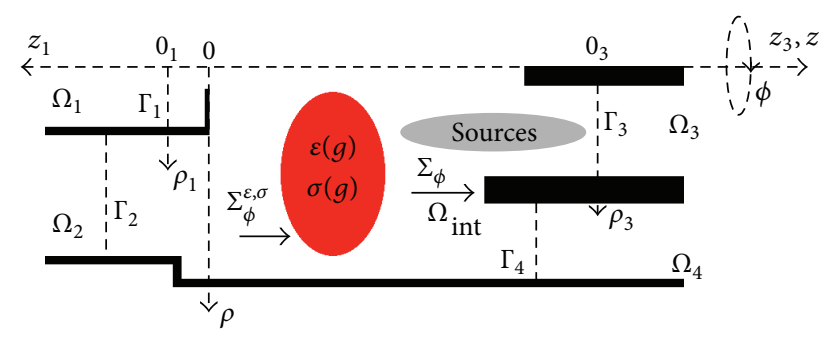

FIGURE 1: Open axially symmetrical waveguide transformer.

$U(g, t)=H_{\phi}(g, t)$ for $\mathrm{TM}_{0}$-waves. The function $U(g, t)$ determines other nonzero field components as well: $H_{\rho}, H_{z}$ in the case of $\mathrm{TE}_{0}$-waves and $E_{\rho}, E_{z}$ in the case of $\mathrm{TM}_{0}$-waves (see, e.g., formulas (1.13) and (1.15) in [3]). By $\Sigma=\Sigma_{\phi} \times[0,2 \pi]$, we denote perfectly conducting surfaces obtained by rotating the piecewise smooth curve $\Sigma_{\phi}$ about the $z$-axis. The relative permittivity $\varepsilon(g)(g=\{\rho, z\})$ and specific conductivity $\sigma(g)$ are smooth enough nonnegative functions inside $\Omega_{\text {int }}$ and take free space values outside (in case of $\mathrm{TE}_{0}$-waves) or $\varepsilon(g) \equiv 1$ and $\sigma(g) \equiv 0$ (in $\mathrm{TM}_{0}$-case), $\eta_{0}=\left(\mu_{0} / \varepsilon_{0}\right)^{1 / 2}$ is the impedance of free space, $\varepsilon_{0}$ and $\mu_{0}$ are the electric and magnetic constants of vacuum. We use the SI system of units. The "time" variable $t$ is the product of the real time by the velocity of light in free space and so is measured in meters.

The domain of analysis $\Omega$ is the part of the half-plane $\Omega_{\text {total }}=\{p=\{\rho, \phi, z\}: \rho>0,|z| \geq 0, \phi=\pi / 2\}$ bounded by the contours $\Sigma_{\phi}$. The regions $\Omega_{\text {int }}$ and $\Omega_{\text {add }}=\cup_{j=1}^{J} \Omega_{j}$ (regular semiinfinite circular and coaxial waveguides) are separated by the virtual boundaries $\Gamma_{j}=\left\{g_{j}=\left\{\rho_{j}, z_{j}\right\} \in \Omega: z_{j}=0\right\}$ and $\Omega=\Omega_{\text {int }} \cup \Omega_{\text {add }} \cup \Gamma_{\text {add }}, \Gamma_{\text {add }}=\cup_{j=1}^{J} \Gamma_{j}$. Here, $\left\{\rho_{j}, \phi_{j}, z_{j}\right\}$ is the local coordinate system associated with the waveguide $\Omega_{j}$ (Figure 1).

The functions $F(g, t), \varphi(g), \psi(g), \sigma(g)$, and $\varepsilon(g)-1$ which are finite in the closure $\bar{\Omega}$ of $\Omega$ are supposed to satisfy the theorem on the unique solvability of problem (4) in the Sobolev space $W_{2}^{1}\left(\Omega^{T}\right), \Omega^{T}=\Omega \times(0, T),(0, T)=\{t: 0<$ $t<T<\infty\}$ (see Statement 2 and $[3,36]$ ). The "current" and "instantaneous" sources given by the functions $F(g, t)$ and $\varphi(g), \psi(g)$ as well as all scattering elements given by the functions $\varepsilon(g), \sigma(g)$, and by the contours $\Sigma_{\phi}$ are located in the region $\Omega_{\text {int }}$. In axially symmetrical problems, at the points $g=\{\rho, z\}$ such that $\rho=0$, only $H_{z^{-}}$or $E_{z}$-field components are nonzero $[2,3,8]$. Hence, it follows that $U(0, z, t)=0$ for $|z|<\infty, t \geq 0$ in (4).

Let us assume that $0<\nu \leq 1 / \varepsilon(g) \leq \mu<\infty$ for $g \in \Omega$ and that the functions $\sigma / \varepsilon, \varepsilon^{\prime} / \varepsilon^{2}$ are bounded in $\Omega$. Then, the following statement (see also $[3,36]$ ) is true.

Statement. Let $F(g, t) / \varepsilon(g) \in L_{2,1}\left(\Omega^{T}\right), \varphi(g) \in \dot{W}_{2}^{1}(\Omega)$ (for $\mathrm{TE}_{0}$-waves) or $\varphi(g) \in W_{2}^{1}(\Omega)$ (for $\mathrm{TM}_{0}$-waves) and $\psi(g) \epsilon$ $L_{2}(\Omega)$. Then, problem (4) has a generalized solution from $W_{2}^{1}\left(\Omega^{T}\right)$, and the uniqueness theorem is true in this space.

Here, the following notation is used: $\varepsilon^{\prime}$ is the partial derivative of $\varepsilon(g)$ with respect to $\rho$ or $z, L_{n}(G)$ is the space of functions $f(g)$ (where $g \in G$ ) for which the function $|f(g)|^{n}$ is integrable in $G, W_{m}^{l}(G)$ is the set of all the elements $f(g)$ from $L_{m}(G)$ having generalized derivatives up to the order $l$ inclusive from $L_{m}(G), L_{2,1}\left(G^{T}\right)$ is the space containing all elements $f(g, t) \in L_{1}\left(G^{T}\right)$ with finite norm $\|f\|=$ $\int_{0}^{T}\left(\int_{G}|f|^{2} d g\right)^{1 / 2} d t$, and $W_{2}^{1}(G)$ is the subspace of space $W_{2}^{1}(G)$, in which the set of finitary and infinitely differentiable in $G$ functions is a dense set.

\section{Exact Radiation Conditions for Outgoing Pulsed Waves}

In the domain $\Omega_{j}$ (Figure 2 ), where the field $U(g, t)$ propagates freely up to $z_{j}=\infty$ as $t \rightarrow \infty$, the $2 \mathrm{D}$ initial boundary value problem (4) can be rewritten in the local coordinates $g_{j}=\left\{\rho_{j}, z_{j}\right\}$ in the following way:

$$
\begin{gathered}
{\left[-\frac{\partial^{2}}{\partial t^{2}}+\frac{\partial^{2}}{\partial z_{j}^{2}}+\frac{\partial}{\partial \rho_{j}}\left(\frac{1}{\rho_{j}} \frac{\partial}{\partial \rho_{j}} \rho_{j}\right)\right] U\left(g_{j}, t\right)=0 ;} \\
t>0, g_{j} \in \Omega_{j}, \\
\left.U\left(g_{j}, t\right)\right|_{t=0}=0,\left.\quad \frac{\partial}{\partial t} U\left(g_{j}, t\right)\right|_{t=0}=0 ; \\
g_{j}=\left\{\rho_{j}, z_{j}\right\} \in \bar{\Omega}_{j}, \\
\left.E_{t g}\left(p_{j}, t\right)\right|_{p_{j}=\left\{\rho_{j}, \phi_{j}, z_{j}\right\} \in \Sigma}=0, \quad U\left(0, z_{j}, t\right)=0 \\
\text { for } 0 \leq z_{j} \leq \infty ; t \geq 0 .
\end{gathered}
$$

Separation of variables in (5) results in

$$
\begin{array}{r}
U\left(g_{j}, t\right)=\sum_{n} u_{n j}\left(z_{j}, t\right) \mu_{n j}\left(\rho_{j}\right), \\
u_{n j}\left(z_{j}, t\right)=\int_{0, \text { or } b_{j}}^{a_{j}} \int U\left(g_{j}, t\right) \mu_{n j}\left(\rho_{j}\right) \rho_{j} d \rho_{j} ; \\
g_{j}=\left\{\rho_{j}, z_{j}\right\} \in \Omega_{j},
\end{array}
$$

where the orthonormal (with the weight factor $\rho_{j}$ ) basic sets $\left\{\mu_{n j}\left(\rho_{j}\right)\right\}_{n}$ (and the sets $\left\{\lambda_{n j}\right\}_{n}$ of the transversal eigenvalues $\lambda_{n j}$ ) are obtained from the homogeneous Sturm-Liouville problems

$$
\begin{gathered}
{\left[\frac{d}{d \rho_{j}} \frac{1}{\rho_{j}} \frac{d}{d \rho_{j}} \rho_{j}+\lambda_{n j}^{2}\right] \mu_{n j}\left(\rho_{j}\right)=0 ; \quad \rho_{j} \in\left(0, a_{j}\right),} \\
\mu_{n j}(0)=\mu_{n j}\left(a_{j}\right)=0 \quad\left(\mathrm{TE}_{0} \text {-waves }\right) \text { or } \\
\mu_{n j}(0)=\left.\frac{d\left(\rho_{j} \mu_{n j}\left(\rho_{j}\right)\right)}{d \rho_{j}}\right|_{\rho_{j}=a_{j}}=0 \quad\left(\mathrm{TM}_{0} \text {-waves }\right)
\end{gathered}
$$




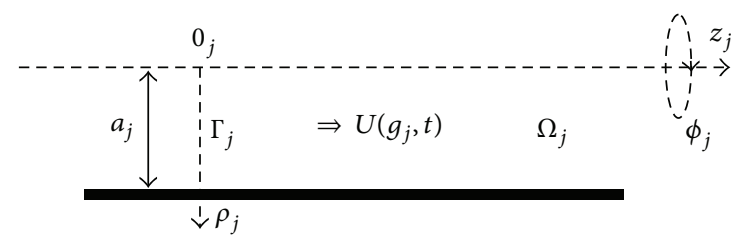

(a)

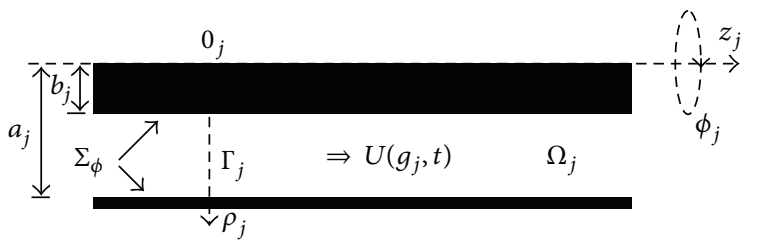

(b)

Figure 2: Virtual boundary $\Gamma_{j}$ in the regular (a) circular and (b) coaxial waveguide $\Omega_{j}$.

(if $\Omega_{j}$ is the circular waveguide with $0 \leq \rho_{j} \leq a_{j}$ ) or

$$
\begin{gathered}
{\left[\frac{d}{d \rho_{j}} \frac{1}{\rho_{j}} \frac{d}{d \rho_{j}} \rho_{j}+\lambda_{n j}^{2}\right] \mu_{n j}\left(\rho_{j}\right)=0 ; \quad \rho_{j} \in\left(b_{j}, a_{j}\right),} \\
\mu_{n j}\left(b_{j}\right)=\mu_{n j}\left(a_{j}\right)=0 \quad\left(\mathrm{TE}_{0} \text {-waves }\right) \text { or } \\
\left.\frac{d\left(\rho_{j} \mu_{n j}\left(\rho_{j}\right)\right)}{d \rho_{j}}\right|_{\rho_{j}=b_{j}}=\left.\frac{d\left(\rho_{j} \mu_{n j}\left(\rho_{j}\right)\right)}{d \rho_{j}}\right|_{\rho_{j}=a_{j}} \\
=0 \quad\left(\mathrm{TM}_{0} \text {-waves }\right)
\end{gathered}
$$

(if $\Omega_{j}$ is the coaxial waveguide with $b_{j} \leq \rho_{j} \leq a_{j}$ ). Hereafter, $n=0,1,2, \ldots$ only in the case of $\mathrm{TM}_{0}$-waves and only for coaxial waveguide $\Omega_{j}$. In all other cases, $n=1,2,3, \ldots$. The form of the boundary conditions on waveguide walls for the case of $\mathrm{TM}_{0}$-waves in (7) and (8) is determined by the following relationships: $U(g, t)=H_{\phi}(g, t),\left.E_{t g}\left(p_{j}, t\right)\right|_{p_{j} \in \Sigma}=$ $\left.E_{z_{j}}\left(p_{j}, t\right)\right|_{p_{j} \in \Sigma}$, and $\partial E_{z_{j}} / \partial t=\eta_{0} \rho_{j}^{-1} \partial\left(\rho_{j} H_{\phi_{j}}\right) / \partial \rho_{j}[3]$.

The spatial-temporal amplitudes $u_{n j}\left(z_{j}, t\right)$ of the wave $U\left(g_{j}, t\right), g_{j} \in \Omega_{j}$ are obtained by solving the initial boundary value problems

$$
\begin{gathered}
{\left[-\frac{\partial^{2}}{\partial t^{2}}+\frac{\partial^{2}}{\partial z_{j}^{2}}-\lambda_{n j}^{2}\right] u_{n j}\left(z_{j}, t\right)=0 ; \quad t>0, z_{j}>0} \\
u_{n j}\left(z_{j}, 0\right)=0,\left.\quad \frac{\partial}{\partial t} u_{n j}\left(z_{j}, t\right)\right|_{t=0}=0 ; \quad z_{j} \geq 0
\end{gathered}
$$

(it is assumed that the excitation $U(g, t)$, being generated by the sources $\varphi(g), \psi(g)$, and $F(g, t)$ located in $\Omega_{\text {int }}$, has not yet reached the boundary $z_{j}=0$ separating the regions $\Omega_{\text {int }}$ and $\Omega_{j}$ by the time $t=0$ ).
Analytical representations for solutions $\mu_{n j}(\rho)$ and $\lambda_{n j}$ to the problems (7) and (8) are well-known $[3,8]$ and, for $\mathrm{TE}_{0^{-}}$ waves, take the form:

$$
\begin{aligned}
\mu_{n j}\left(\rho_{j}\right)= & G_{1}\left(\lambda_{n j}, \rho_{j}\right) \sqrt{2} \\
& \times\left[a_{j}^{2} G_{0}^{2}\left(\lambda_{n j}, a_{j}\right)-b_{j}^{2} G_{0}^{2}\left(\lambda_{n j}, b_{j}\right)\right]^{-1 / 2} ; \\
& b_{j}<\rho_{j}<a_{j},
\end{aligned}
$$

$\lambda_{n j}>0$ are the roots of the equation $G_{1}\left(\lambda_{j}, a_{j}\right)=0$,

$$
G_{q}\left(\lambda_{j}, \rho_{j}\right)=J_{q}\left(\lambda_{j} \rho_{j}\right) N_{1}\left(\lambda_{j} b_{j}\right)-N_{q}\left(\lambda_{j} \rho_{j}\right) J_{1}\left(\lambda_{j} b_{j}\right) ;
$$

for coaxial waveguide $\Omega_{j}$ and

$$
\begin{array}{r}
\mu_{n j}\left(\rho_{j}\right)=J_{1}\left(\lambda_{n j} \rho_{j}\right) \sqrt{2}\left[a_{j} J_{0}\left(\lambda_{n j} a_{j}\right)\right]^{-1} ; \\
0<\rho_{j}<a_{j},
\end{array}
$$

$\lambda_{n j}>0$ are the roots of the equation $J_{1}\left(\lambda_{j} a_{j}\right)=0$ for circular waveguide $\Omega_{j}$. For $\mathrm{TM}_{0}$-waves, we have

$$
\begin{array}{r}
\mu_{n j}\left(\rho_{j}\right)=J_{1}\left(\lambda_{n j} \rho_{j}\right) \sqrt{2}\left[a_{j} J_{1}\left(\lambda_{n j} a_{j}\right)\right]^{-1} ; \\
0<\rho_{j}<a_{j},
\end{array}
$$

$\lambda_{n j}>0$ are the roots of the equation $J_{0}\left(\lambda_{j} a_{j}\right)=0$

(circular waveguide $\Omega_{j}$ ) and

$$
\begin{aligned}
& \mu_{n j}\left(\rho_{j}\right)= \widetilde{G}_{1}\left(\lambda_{n j}, \rho_{j}\right) \sqrt{2} \\
& \times\left[a_{j}^{2} \widetilde{G}_{1}^{2}\left(\lambda_{n j}, a_{j}\right)-b_{j}^{2} \widetilde{G}_{1}^{2}\left(\lambda_{n j}, b_{j}\right)\right]^{-1 / 2} \\
& \text { for } n=1,2, \ldots, \\
& \mu_{0}\left(\rho_{j}\right)=\left[\rho_{j} \sqrt{\ln \left(a_{j} / b_{j}\right)}\right]^{-1} ; \quad b_{j}<\rho_{j}<a_{j},
\end{aligned}
$$

$\lambda_{n j}>0$ for $n=1,2, \ldots$ are the roots of the equation

$$
\begin{gathered}
\widetilde{G}_{0}\left(\lambda_{j}, b_{j}\right)=0, \quad \lambda_{0 j}=0, \\
\widetilde{G}_{q}\left(\lambda_{j}, \rho_{j}\right)=J_{q}\left(\lambda_{j} \rho_{j}\right) N_{0}\left(\lambda_{j} a_{j}\right)-N_{q}\left(\lambda_{j} \rho_{j}\right) J_{0}\left(\lambda_{j} a_{j}\right) ; \\
q=0,1
\end{gathered}
$$

(coaxial waveguide $\Omega_{j}$ ). Here, $J_{q}(\cdots)$ and $N_{q}(\cdots)$ are the Bessel and Neumann cylindrical functions.

By applying to (9) the cosine Fourier transform

$$
\begin{aligned}
\tilde{f}(\omega)= & \sqrt{\frac{2}{\pi}} \int_{0}^{\infty} f\left(z_{j}\right) \cos \left(\omega z_{j}\right) d z_{j} \\
& \longleftrightarrow f\left(z_{j}\right)=\sqrt{\frac{2}{\pi}} \int_{0}^{\infty} \tilde{f}(\omega) \cos \left(\omega z_{j}\right) d \omega
\end{aligned}
$$


and taking into account that

$$
-\omega^{2} \tilde{f}(\omega)-\left.\sqrt{\frac{2}{\pi}}\left[\frac{d}{d z_{j}} f\left(z_{j}\right)\right]\right|_{z_{j}=0} \longleftrightarrow \frac{d^{2}}{d z_{j}^{2}} f\left(z_{j}\right)
$$

[37], we are led to the following Cauchy problems for the images $\widetilde{u}_{n j}(\omega, t)$ of the functions $u_{n j}\left(z_{j}, t\right)$ :

$$
\begin{array}{r}
D\left(\sqrt{\lambda_{n j}^{2}+\omega^{2}}\right)\left[\widetilde{u}_{n j}(\omega, t)\right] \equiv\left[\frac{\partial^{2}}{\partial t^{2}}+\left(\lambda_{n j}^{2}+\omega^{2}\right)\right] \widetilde{u}_{n j}(\omega, t) \\
=-\sqrt{\frac{2}{\pi}} u_{n j}^{\prime}(0, t) ; \quad \omega>0, t>0, \\
\tilde{u}_{n j}(\omega, 0)=0,\left.\quad \frac{\partial}{\partial t} \widetilde{u}_{n j}(\omega, t)\right|_{t=0}=0 ; \quad \omega \geq 0,
\end{array}
$$

where $u_{n j}^{\prime}(c, t)=\partial u_{n j}\left(z_{j}, t\right) /\left.\partial z_{j}\right|_{z_{j}=c}$. The fact that the wave $U\left(g_{j}, t\right)\left(g_{j} \in \Omega_{j}\right)$ is an outgoing one and thus the amplitudes $u_{n j}\left(z_{j}, t\right)$ of all its partial components are zero at any time $t$ for the values of $z_{j}$ large enough played an important part in derivation of (16).

Continuing the functions $\tilde{u}_{n j}(\omega, t)$ and $u_{n j}^{\prime}(0, t)$ by zero on the semiaxis $t<0$, we pass on to generalized formulation of the Cauchy problems (16) [38]:

$$
\begin{aligned}
& D\left(\sqrt{\lambda_{n j}^{2}+\omega^{2}}\right)\left[\tilde{u}_{n j}(\omega, t)\right] \\
& =-\sqrt{\frac{2}{\pi}} u_{n}^{\prime}(0, t)+\delta^{(1)}(t) \widetilde{u}_{n j}(\omega, 0) \\
& +\left.\delta(t) \frac{\partial}{\partial t} \tilde{u}_{n j}(\omega, t)\right|_{t=0}=-\sqrt{\frac{2}{\pi}} u_{n j}^{\prime}(0, t) ; \\
& \omega>0, \quad-\infty<t<\infty,
\end{aligned}
$$

where $\delta(\cdots)$ and $\delta^{(1)}(\cdots)$ are the Dirac delta-function and its generalized derivative of the first order.

A convolution of the fundamental solution $G(\lambda, t)=$ $\chi(t) \lambda^{-1} \sin \lambda t(\chi(\cdots)$ is the Heaviside step function) of the operator $D(\lambda)[\cdots]$ with the right-hand side of the equation $D(\lambda)[u(t)]=f(t)$ gives its solution $u(t)[3,38]$. Therefore, the unknown functions $\widetilde{u}_{n j}(\omega, t)$ can be represented as

$$
\begin{array}{r}
\tilde{u}_{n j}(\omega, t) \\
=-\sqrt{\frac{2}{\pi}} \int_{0}^{t} \sin \left[(t-\tau) \sqrt{\lambda_{n j}^{2}+\omega^{2}}\right] \frac{u_{n j}^{\prime}(0, \tau)}{\sqrt{\lambda_{n j}^{2}+\omega^{2}}} d \tau \\
\omega \geq 0, \quad t \geq 0 .
\end{array}
$$

Applying to (18) the inverse transform (14), we get for the originals $u_{n j}\left(z_{j}, t\right)$ :

$$
\begin{aligned}
u_{n j}\left(z_{j}, t\right) & \\
= & -\int_{0} J_{0}\left[\lambda_{n j}\left((t-\tau)^{2}-z_{j}^{2}\right)^{1 / 2}\right] \\
& \times \chi\left[(t-\tau)-z_{j}\right] u_{n j}^{\prime}(0, \tau) d \tau ; \quad z_{j} \geq 0, t \geq 0 .
\end{aligned}
$$

On multiplication of (19) by $\mu_{n j}\left(\rho_{j}\right)$ and summation over all $n$, we obtain the exact radiation condition (ERC) for the pulsed waves $U\left(g_{j}, t\right)$ outgoing towards $z_{j}=\infty$ :

$$
\begin{aligned}
& U\left(g_{j}, t\right) \\
& =-\sum_{n}\left\{\int_{0}^{t-z_{j}} J_{0}\left[\lambda_{n j}\left((t-\tau)^{2}-z_{j}^{2}\right)^{1 / 2}\right]\right. \\
& \left.\times\left[\left.\int_{\rho_{j(1)}}^{\rho_{j(2)}} \frac{\partial U\left(\rho_{j}, z_{j}, \tau\right)}{\partial z_{j}}\right|_{z_{j}=0} \mu_{n j}\left(\rho_{j}\right) \rho_{j} d \rho_{j}\right] d \tau\right\} \\
& \quad \times \mu_{n j}\left(\rho_{j}\right) ; \quad g_{j}=\left\{\rho_{j}, z_{j}\right\} \in \Omega_{j}, t \geq z_{j} .
\end{aligned}
$$

In (20), one should set $\rho_{j(1)}=0$ and $\rho_{j(2)}=a_{j}$ for a circular waveguide $\Omega_{j}$ or $\rho_{j(1)}=b_{j}$ and $\rho_{j(2)}=a_{j}$ for a coaxial waveguide.

Equations (19) and (20) specify the diagonal transport operator $X_{0 \rightarrow z_{j}}(t)\left[u_{j}^{\prime}\right]$, which operates in the space of amplitudes $u_{j}\left(z_{j}, t\right)=\left\{u_{n j}\left(z_{j}, t\right)\right\}_{n}$ according to the rule

$$
\begin{array}{r}
u_{j}\left(z_{j}, t\right)=X_{0 \rightarrow z_{j}}(t)\left[u_{j}^{\prime}(0, \tau)\right] ; \quad u_{j}^{\prime}(c, \tau)=\left\{u_{n j}^{\prime}(c, \tau)\right\}_{n}, \\
z_{j} \geq 0, t \geq z_{j}, t-z_{j} \geq \tau \geq 0,
\end{array}
$$

and the operator

$$
\begin{gathered}
U\left(g_{j}, t\right)=Z_{q_{j} \in \Gamma_{j} \rightarrow g_{j} \in \Omega_{j}}(t)\left[U^{\prime}\left(q_{j}, \tau\right)\right] \\
U^{\prime}\left(q_{j}, \tau\right)=\left.\frac{\partial U\left(g_{j}, \tau\right)}{\partial z_{j}}\right|_{z_{j}=0}, \\
t \geq z_{j}, \quad t-z_{j} \geq \tau \geq 0
\end{gathered}
$$

accounting for all transformations of the wave $U\left(g_{j}, t\right)$ outgoing through the boundary $\Gamma_{j}$ into the hollow regular waveguide $\Omega_{j}$.

\section{Nonlocal Absorbing Conditions}

By dropping the observation point in (19) onto the artificial boundary $\Gamma_{j}\left(z_{j}=0\right)$, we obtain

$$
u_{n j}(0, t)=-\int_{0}^{t} J_{0}\left[\lambda_{n j}(t-\tau)\right] u_{n j}^{\prime}(0, \tau) d \tau ; \quad t \geq 0 .
$$

Differentiating (23) with respect to $t$, we can write

$$
\begin{aligned}
& {\left.\left[\frac{\partial}{\partial t}+\frac{\partial}{\partial z_{j}}\right] u_{n j}\left(z_{j}, t\right)\right|_{z_{j}=0}} \\
& \quad=\lambda_{n j} \int_{0}^{t} J_{1}\left[\lambda_{n j}(t-\tau)\right] u_{n j}^{\prime}(0, \tau) d \tau ; \quad t \geq 0 .
\end{aligned}
$$


Here, the well-known relationships $d J_{0}(x) / d x=-J_{1}(x)$, $J_{0}(0)=1$, and $\chi^{(1)}(t-\tau)=\delta(t-\tau)$, where $\chi^{(1)}(\cdots)$ stands for the generalized derivative of $\chi(\cdots)$, have been used.

Let us now apply to (24) the Laplace transform

$$
\tilde{f}(s)=\int_{0}^{\infty} f(t) \mathrm{e}^{-s t} d t \longleftrightarrow f(t)=\frac{1}{2 \pi i} \int_{\alpha-i \infty}^{\alpha+i \infty} \tilde{f}(s) \mathrm{e}^{s t} d s .
$$

Taking into account the formulas $\tilde{f}_{1}(s) \tilde{f}_{2}(s) \leftrightarrow \int_{0}^{t} f_{1}(t-$

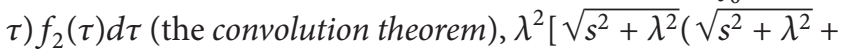
$s)]^{-1} \leftrightarrow \lambda J_{1}(\lambda t)$ [39], and $s \widetilde{f}(s)-f(0) \leftrightarrow d f(t) / d t$, we pass on to the following expression in the space of images $\widetilde{u}_{n j}(z, s)$ :

$$
\left.\left[\frac{\partial}{\partial z_{j}}+s\right] \widetilde{u}_{n j}\left(z_{j}, s\right)\right|_{z_{j}=0}=\frac{\lambda_{n}^{2} \tilde{u}_{n j}^{\prime}(0, s)}{\sqrt{s^{2}+\lambda_{n j}^{2}}\left(\sqrt{s^{2}+\lambda_{n j}^{2}}+s\right)} .
$$

Equation (26) can be transformed to

$$
\widetilde{u}_{n j}^{\prime}(0, s)=-\left(s+\frac{\lambda_{n j}^{2}}{s+\sqrt{s^{2}+\lambda_{n j}^{2}}}\right) \widetilde{u}_{n j}(0, s)
$$

By applying the inverse Laplace transform to (27), we turn back to the originals:

$$
\begin{array}{r}
{\left.\left[\frac{\partial}{\partial t}+\frac{\partial}{\partial z_{j}}\right] u_{n}\left(z_{j}, t\right)\right|_{z_{j}=0}} \\
=-\lambda_{n j} \int_{0}^{t} J_{1}\left[\lambda_{n j}(t-\tau)\right](t-\tau)^{-1} u_{n j}(0, \tau) d \tau ; \\
t \geq 0 .
\end{array}
$$

Here, the formula $\left(s+\sqrt{s^{2}+\lambda^{2}}\right)^{-1} \leftrightarrow(\lambda t)^{-1} J_{1}(\lambda t)[40]$ is used.

To justify the use of the Laplace transform (25) when passing from (24) to (28), we refer to the estimates performed in the book [41]. From these estimates, it follows that, at points $g$ of any bounded subdomain of the domain $\Omega$, the field $U(g, t)$ generated by a system of sources with compact supports cannot grow faster than $\exp (\alpha t)$ for $t \rightarrow \infty$, where $\alpha>0$ is some constant. The estimates are valid for all electrodynamic structures whose spectrum of complexvalued eigenfrequencies $\left\{\bar{k}_{n}\right\}_{n}$ does not contain the points $\bar{k}_{m}$ from the upper half-plane of the first (physical) sheet, which constitutes a natural domain of variation of the complex frequency parameter $k$ [3]. All the open structures considered in this section fall into this category [42].
Rewriting (23), (24), and (28) in terms of (6), we obtain

$$
\begin{aligned}
& U\left(\rho_{j}, 0, t\right) \\
& =-\sum_{n}\left\{\int_{0}^{t} J_{0}\left[\lambda_{n j}(t-\tau)\right]\right. \\
& \left.\times\left[\left.\int_{\rho_{j(1)}}^{\rho_{j(2)}} \frac{\partial U\left(\rho_{j}, z_{j}, \tau\right)}{\partial z_{j}}\right|_{z_{j}=0} \mu_{n j}\left(\rho_{j}\right) \rho_{j} d \rho_{j}\right] d \tau\right\} \\
& \times \mu_{n j}\left(\rho_{j}\right) ; \quad \rho_{j(1)} \leq \rho \leq \rho_{j(2)}, t \geq 0,
\end{aligned}
$$

$$
\begin{aligned}
& {\left.\left[\frac{\partial}{\partial t}+\frac{\partial}{\partial z_{j}}\right] U\left(\rho_{j}, z_{j}, t\right)\right|_{z_{j}=0}} \\
& =\sum_{n} \lambda_{n j}\left\{\int_{0}^{t} J_{1}\left[\lambda_{n j}(t-\tau)\right] \times\left[\left.\int_{\rho_{j(1)}}^{\rho_{j(2)}} \frac{\partial U\left(\rho_{j}, z_{j}, \tau\right)}{\partial z_{j}}\right|_{z_{j}=0}\right.\right. \\
& \left.\left.\times \mu_{n j}\left(\rho_{j}\right) \rho_{j} d \rho_{j}\right] d \tau\right\} \\
& \quad \times \mu_{n j}\left(\rho_{j}\right) ; \quad \rho_{j(1)} \leq \rho_{j} \leq \rho_{j(2)}, t \geq 0,
\end{aligned}
$$

$$
\begin{aligned}
& {\left.\left[\frac{\partial}{\partial t}+\frac{\partial}{\partial z_{j}}\right] U\left(\rho_{j}, z_{j}, t\right)\right|_{z_{j}=0}} \\
& =-\sum_{n} \lambda_{n j}\left\{\int_{0}^{t} J_{1}\left[\lambda_{n j}(t-\tau)\right] \times(t-\tau)^{-1}\right. \\
& \left.\times\left[\int_{\rho_{j(1)}}^{\rho_{j(2)}} U\left(\rho_{j}, 0, \tau\right) \mu_{n j}\left(\rho_{j}\right) \rho_{j} d \rho_{j}\right] d \tau\right\} \\
& \quad \times \mu_{n j}\left(\rho_{j}\right) ; \quad \rho_{j(1)} \leq \rho_{j} \leq \rho_{j(2)}, t \geq 0 .
\end{aligned}
$$

Relationships (23), (24), (28), and (29)-(31) are valid for all the waves $U\left(\rho_{j}, z_{j}, t\right)$ outgoing into the waveguide $\Omega_{j}$. Equations (23), (24), and (28) relate space-time amplitudes of all modes guided by the regular structure towards $z_{j}=\infty$ on the boundary $\Gamma_{j}$, while (29)-(31) relate space-time amplitudes of the waves integrally. Therefore, the open problem (4) and the problem with a bounded domain of analysis $\Omega_{\text {int }}$ given by (4) together with any boundary condition like (29)-(31) on the virtual boundaries $\Gamma_{j}, j=1,2, \ldots, J$, are equivalent (see Section 6). For the same reason, the boundary conditions given by (29)-(31) can be considered as exact boundary conditions: the wave $U(g, t)$ is not distorted when crossing the boundary $\Gamma_{j}$; there is no reflection into the domain $\Omega_{\text {int }}$; the wave $U(g, t)$ passes into the domain $\Omega_{j}$ completely, as if it is absorbed by that domain or by its boundary $\Gamma_{j}$. For the first time, (23) and (29) were used as exact absorbing boundary conditions in [1]. 
Conditions (29)-(31) are nonlocal in the spatial variable $\left(\rho_{j}\right)$ and in time as well; the function $U\left(\rho_{j}, z_{j}, t\right)$ and its derivatives at each point of the boundary $z_{j}=0$ and at each time point $t$ are related to the values of this function or its derivatives at all points of the boundary and at all time points $\tau<t$.

\section{Local Absorbing Conditions}

By using the relation

$$
J_{0}(x)=\frac{2}{\pi} \int_{0}^{\pi / 2} \cos (x \sin \varphi) d \varphi
$$

[37], rewrite (23) as

$$
\begin{array}{r}
u_{n j}(0, t) \\
=-\frac{2}{\pi} \int_{0}^{\pi / 2}\left\{\int_{0}^{t} \cos \left[\lambda_{n j}(t-\tau) \sin \varphi\right] u_{n j}^{\prime}(0, \tau) d \tau\right\} d \varphi \\
t \geq 0 .
\end{array}
$$

Denote

$$
\begin{array}{r}
w_{n j}(t, \varphi)=-\int_{0}^{t} \frac{\sin \left[\lambda_{n j}(t-\tau) \sin \varphi\right] u_{n j}^{\prime}(0, \tau)}{\lambda_{n j} \sin \varphi} d \tau ; \\
t \geq 0, \quad 0 \leq \varphi \leq \frac{\pi}{2} .
\end{array}
$$

Then,

$$
\frac{\partial w_{n j}(t, \varphi)}{\partial t}=-\int_{0}^{t} \cos \left[\lambda_{n j}(t-\tau) \sin \varphi\right] u_{n j}^{\prime}(0, \tau) d \tau,
$$

and we have from (33) that

$$
u_{n j}(0, t)=\frac{2}{\pi} \int_{0}^{\pi / 2} \frac{\partial w_{n j}(t, \varphi)}{\partial t} d \varphi ; \quad t \geq 0
$$

The integral form (34) is equivalent to the following differential form:

$$
\begin{gathered}
{\left[\frac{\partial^{2}}{\partial t^{2}}+\lambda_{n j}^{2} \sin ^{2} \varphi\right] w_{n j}(t, \varphi)=-u_{n j}^{\prime}(0, t)} \\
t>0 \\
w_{n j}(0, \varphi)=\left.\frac{\partial w_{n j}(t, \varphi)}{\partial t}\right|_{t=0}=0
\end{gathered}
$$

Indeed, by passing on to the generalized formulation of the corresponding Cauchy problem and using the fundamental solution $G(\lambda, t)=\chi(t) \lambda^{-1} \sin \lambda t$ of the operator $D(\lambda) \equiv$ $\left[d^{2} / d t^{2}+\lambda^{2}\right]$ (see [3]), we can easily verify that formulas (34) and (37) determine the same function $w_{n j}(t, \varphi)$.
Let us now multiply (36) and (37) by $\mu_{n j}\left(\rho_{j}\right)$ and sum over all $n$. As a result, taking into consideration that

$$
\begin{aligned}
& \sum_{n} \lambda_{n j}^{2} u_{n j}\left(z_{j}, t\right) \mu_{n j}\left(\rho_{j}\right)=-\frac{\partial}{\partial \rho_{j}} \frac{1}{\rho_{j}} \frac{\partial}{\partial \rho_{j}} \rho_{j} U\left(\rho_{j}, z_{j}, t\right), \\
& \sum_{n} \lambda_{n j}^{2} w_{n j}(t, \varphi) \mu_{n j}\left(\rho_{j}\right)=-\frac{\partial}{\partial \rho_{j}} \frac{1}{\rho_{j}} \frac{\partial}{\partial \rho_{j}} \rho_{j} W\left(\rho_{j}, t, \varphi\right)
\end{aligned}
$$

for

$$
W\left(\rho_{j}, t, \varphi\right)=\sum_{n} w_{n j}(t, \varphi) \mu_{n j}\left(\rho_{j}\right)
$$

(see problems (7) and (8)), we obtain

$$
\begin{array}{r}
U\left(\rho_{j}, 0, t\right)=\frac{2}{\pi} \int_{0}^{\pi / 2} \frac{\partial W\left(\rho_{j}, t, \varphi\right)}{\partial t} d \varphi \\
t \geq 0, \quad \rho_{j(1)} \leq \rho_{j} \leq \rho_{j(2)},
\end{array}
$$

$$
\left[\frac{\partial^{2}}{\partial t^{2}}-\sin ^{2} \varphi \frac{\partial}{\partial \rho_{j}} \frac{1}{\rho_{j}} \frac{\partial}{\partial \rho_{j}} \rho_{j}\right] W\left(\rho_{j}, t, \varphi\right)
$$

$$
\begin{array}{r}
=-\left.\frac{\partial U\left(\rho_{j}, z_{j}, t\right)}{\partial z_{j}}\right|_{z_{j}=0} ; \quad \rho_{j(1)}<\rho_{j}<\rho_{j(2)}, t>0, \\
W\left(\rho_{j}, 0, \varphi\right)=\left.\frac{\partial W\left(\rho_{j}, t, \varphi\right)}{\partial t}\right|_{t=0}=0, \quad \rho_{j(1)} \leq \rho_{j} \leq \rho_{j(2)} .
\end{array}
$$

Here, $W\left(\rho_{j}, t, \varphi\right)$ is an auxiliary function. It can be determined by solving the initial boundary value problem (40b), which is the inner problem with respect to the local EAC (40a); $0 \leq \varphi \leq \pi / 2$ is a numerical parameter.

Transforming (24) and (28) in a similar way, we obtain the following exact local boundary conditions, differing from (40a) and (40b):

$$
\begin{array}{r}
{\left.\left[\frac{\partial}{\partial t}+\frac{\partial}{\partial z_{j}}\right] U\left(\rho_{j}, z_{j}, t\right)\right|_{z_{j}=0}=\frac{2}{\pi} \int_{0}^{\pi / 2} W\left(\rho_{j}, t, \varphi\right) \cos ^{2} \varphi d \varphi ;} \\
t \geq 0, \rho_{j(1)} \leq \rho_{j} \leq \rho_{j(2)},
\end{array}
$$

$$
\begin{aligned}
& {\left[\frac{\partial^{2}}{\partial t^{2}}-\cos ^{2} \varphi \frac{\partial}{\partial \rho_{j}} \frac{1}{\rho_{j}} \frac{\partial}{\partial \rho_{j}} \rho_{j}\right] W\left(\rho_{j}, t, \varphi\right)=-\frac{\partial}{\partial \rho_{j}} \frac{1}{\rho_{j}} \frac{\partial}{\partial \rho_{j}} \rho_{j}} \\
& \quad \times\left[\left.\frac{\partial}{\partial z_{j}} U\left(\rho_{j}, z_{j}, t\right)\right|_{z_{j}=0}\right] ; \quad \rho_{j(1)}<\rho_{j}<\rho_{j(2)}, t>0, \\
& W\left(\rho_{j}, 0, \varphi\right)=\left.\frac{\partial W\left(\rho_{j}, t, \varphi\right)}{\partial t}\right|_{t=0}=0 ; \quad \rho_{j(1)} \leq \rho_{j} \leq \rho_{j(2)},
\end{aligned}
$$




$$
\begin{gathered}
{\left.\left[\frac{\partial}{\partial t}+\frac{\partial}{\partial z_{j}}\right] U\left(\rho_{j}, z_{j}, t\right)\right|_{z_{j}=0}} \\
=\frac{2}{\pi} \int_{0}^{\pi / 2} \frac{\partial W\left(\rho_{j}, t, \varphi\right)}{\partial t} \sin ^{2} \varphi d \varphi ; \\
t \geq 0, \quad \rho_{j(1)} \leq \rho_{j} \leq \rho_{j(2)}, \\
\left.=\frac{\partial}{\partial \rho_{j}} \frac{1}{\rho_{j}} \frac{\partial}{\partial \rho_{j}} \rho_{j} U\left(\rho_{j}, 0, t\right) ; \quad \rho_{j(1)}^{2} \varphi \frac{\partial}{\partial \rho_{j}} \frac{1}{\rho_{j}} \frac{\partial}{\partial \rho_{j}} \rho_{j}\right] W\left(\rho_{j}, t, \varphi\right) \\
W\left(\rho_{j}, 0, \varphi\right)=\left.\frac{\partial W\left(\rho_{j(2)}, t>0\right.}{\partial t}\right|_{t=0}=0 ; \quad \rho_{j(1)} \leq \rho_{j} \leq \rho_{j(2)} .
\end{gathered}
$$

When deriving (41a) and (41b), the following formula was used:

$$
J_{1}(x)=\frac{2}{\pi} \int_{0}^{\pi / 2} \sin (x \cos \varphi) \cos \varphi d \varphi
$$

[43], along with the substitutions

$$
\begin{gathered}
w_{n j}(t, \varphi)=\lambda_{n j} \int_{0}^{t} \frac{\sin \left[\lambda_{n j}(t-\tau) \cos \varphi\right] u_{n j}^{\prime}(0, \tau)}{\cos \varphi} d \tau ; \\
t \geq 0,0 \leq \varphi \leq \frac{\pi}{2},
\end{gathered}
$$

while, in derivation of (42a) and (42b), we applied the integral Poisson formula

$$
J_{1}(x)=\frac{2 x}{\pi} \int_{0}^{\pi / 2} \cos (x \cos \varphi) \sin ^{2} \varphi d \varphi
$$

[37] and

$$
\begin{array}{r}
w_{n j}(t, \varphi)=-\lambda_{n j} \int_{0}^{t} \frac{\sin \left[\lambda_{n j}(t-\tau) \cos \varphi\right] u_{n j}(0, \tau)}{\cos \varphi} d \tau ; \\
t \geq 0,0 \leq \varphi \leq \frac{\pi}{2} .
\end{array}
$$

One should supplement the initial boundary value problems given by (40b), (41b), and (42b) in (40a)-(42b) and posed relative to the auxiliary functions $W\left(\rho_{j}, t, \varphi\right)$, by the following boundary conditions for all time points $t \geq 0$ :

$$
\begin{gathered}
W(0, t, \varphi)=W\left(a_{j}, t, \varphi\right)=0 ; \quad \mathrm{TE}_{0} \text {-waves, } \\
W(0, t, \varphi)=\left.\frac{\partial\left(\rho_{j} W\left(\rho_{j}, t, \varphi\right)\right)}{\partial \rho_{j}}\right|_{\rho_{j}=a_{j}}=0 ; \quad \mathrm{TM}_{0} \text {-waves }
\end{gathered}
$$

(for the domain $\Omega_{j}$ corresponding to a circular waveguide) and

$$
\begin{gathered}
W\left(b_{j}, t, \varphi\right)=W\left(a_{j}, t, \varphi\right)=0 ; \quad \mathrm{TE}_{0} \text {-waves, } \\
\left.\frac{\partial\left(\rho_{j} W\left(\rho_{j}, t, \varphi\right)\right)}{\partial \rho_{j}}\right|_{\rho_{j}=b_{j}}=\left.\frac{\partial\left(\rho_{j} W\left(\rho_{j}, t, \varphi\right)\right)}{\partial \rho_{j}}\right|_{\rho_{j}=a_{j}}=0 ;
\end{gathered}
$$

$\mathrm{TM}_{0}$-waves

(for the domain $\Omega_{j}$ corresponding to a coaxial waveguide).

Formulas (40a)-(42b) are derived from the same equations (23), (24), and (28) as formulas (29)-(31). They, as well as formulas (29)-(31), faithfully describe behavior of the wave $U(g, t)$ on the boundary $\Gamma_{j}$, which is why they also can be used as the exact absorbing conditions for the outgoing pulsed waves formed by the unit. The conditions given by (40a)(42b) differ from those given by (29)-(31) in that they are local: in (40a), (41a), and (42a), the function $U\left(g_{j}, t\right)$ and its derivatives at each fixed point $\rho_{j}$ of the boundary $z_{j}=0$ and at each fixed time point $t$ are determined by the auxiliary function $W\left(\rho_{j}, t, \varphi\right)$ at the same point $\rho_{j}$ and the same time $t$.

\section{Equivalence Theorem}

The exact absorbing conditions derived in Sections 4 and 5 allow us to replace the original open problem (4) by the closed (modified) problem

$$
\begin{gathered}
{\left[-\varepsilon(g) \frac{\partial^{2}}{\partial t^{2}}-\sigma(g) \eta_{0} \frac{\partial}{\partial t}+\frac{\partial^{2}}{\partial z^{2}}+\frac{\partial}{\partial \rho}\left(\frac{1}{\rho} \frac{\partial}{\partial \rho} \rho\right)\right]} \\
\times U(g, t)=F(g, t) ; \quad t>0, g \in \Omega_{\text {int }}, \\
\left.U(g, t)\right|_{t=0}=\varphi(g),\left.\quad \frac{\partial}{\partial t} U(g, t)\right|_{t=0}=\psi(g) ; \\
g=\{\rho, z\} \in \bar{\Omega}_{\text {int }}, \\
\left.E_{t g}(p, t)\right|_{p=\{\rho, \phi, z\} \in \Sigma}=0, \quad U(0, z, t)=0 \\
\quad \text { for }\{0, z\} \in \bar{\Omega}_{\text {int }}, \\
\left.D_{\text {add }}[U(g, t)]\right|_{g \in \Gamma_{\text {add }}}=0 ; \quad t \geq 0
\end{gathered}
$$

together with the total set $(j=1,2, \ldots, J)$ of the exact radiation conditions (20) for the points $g \in \Omega_{\text {add }}=$ $\cup_{j=1}^{J} \Omega_{j}$. Below, we prove that this replacement is equivalent, that is, any solution to the problem (4) is at the same time the solution to the problem (49), (20), and vice versa. By $\left.D_{\text {add }}[U(g, t)]\right|_{g \in \Gamma_{\text {add }}}=0, \Gamma_{\text {add }}=\cup_{j=1}^{J} \Gamma_{j}$, we denote the total set $(j=1,2, \ldots, J)$ of the EAC including any $J$ local or/and nonlocal boundary conditions from (29)-(31) and (40a)(42b).

Problem (4) is uniquely solvable in the space of generalized functions $W_{2}^{1}\left(\Omega^{T}\right)$. Its unique solution $U(g, t)$ is at the same time a solution to problem (49) from the Sobolev 
space $W_{2}^{1}\left(\Omega_{\text {int }}^{T}\right)$, where $\Omega_{\text {int }}^{T}=\Omega_{\text {int }} \times(0, T)$. This direct inclusion is trivial. It is proved by the constructions from Sections 3-5. The inverse inclusion will also be true if only the generalized solution $U(g, t)$ of problem (49) belonging to the space $W_{2}^{1}\left(\Omega_{\text {int }}^{T}\right)$ is unique. Let us prove the uniqueness.

According to [36], the generalized solution of problem (49) is an element $U(g, t)$ of the space $W_{2}^{1}\left(\Omega_{\text {int }}^{T}\right)$ being equal to $\varphi(g)$ at $t=0$ and satisfying the identity

$$
\begin{aligned}
& \int_{\Omega_{\mathrm{int}}^{T}}\left[\varepsilon \frac{\partial U}{\partial t} \frac{\partial \gamma}{\partial t}-\left(\frac{1}{\rho^{2}} \frac{\partial}{\partial \rho} \rho U\right) \frac{\partial(\rho \gamma)}{\partial \rho}\right. \\
& \left.\quad-\frac{\partial U}{\partial z} \frac{\partial \gamma}{\partial z}-\sigma \eta_{0} \frac{\partial U}{\partial t} \gamma\right] d g d t \\
& +\int_{\Phi^{T}}\left[\left(\frac{1}{\rho} \frac{\partial}{\partial \rho} \rho U\right) \gamma \cos (\vec{n}, \vec{\rho})+\frac{\partial U}{\partial z} \gamma \cos (\vec{n}, \vec{z})\right] d s d t \\
& +\int_{\Omega_{\mathrm{int}}} \varepsilon \psi \gamma(g, 0) d g=\int_{\Omega_{\mathrm{int}}^{T}} F \gamma d g d t
\end{aligned}
$$

for any function $\gamma(g, t)$ from $W_{2}^{1}\left(\Omega_{\text {int }}^{T}\right)$ that is zero at $t=T$. Here, $\Phi^{T}$ is a lateral surface of the cylinder $\Omega_{\text {int }}^{T}\left(\Phi^{T}=\Phi \times\right.$ $(0, T) ; \Phi$ is the boundary of the domain $\left.\Omega_{\text {int }}\right) ; \cos (\vec{n}, \vec{\rho})$ and $\cos (\vec{n}, \vec{z})$ are cosines of the angles between the outer normal $\vec{n}$ to the surface $\Phi^{T}$ and the axes $\vec{\rho}$ and $\vec{z}$, respectively. An element of the end surfaces of the cylinder is $d g=\rho d \rho d z$. Identity (50) is derived by multiplying the telegraph equation from (49) by $\gamma(g, t)$ and by integrating the result by parts in $\Omega_{\text {int }}^{T}[36,44]$.

Suppose there exist two solutions of problem (49) belonging to the space $W_{2}^{1}\left(\Omega_{\text {int }}^{T}\right): U_{1}(g, t)$ and $U_{2}(g, t)$. The difference of these solutions $u(g, t)=U_{1}(g, t)-U_{2}(g, t)$ is the solution of the homogeneous problem (49); therefore it satisfies the identity (see formula (50))

$$
\begin{aligned}
\int_{\Omega_{\mathrm{int}}^{T}} & {\left[\varepsilon \frac{\partial u}{\partial t} \frac{\partial \gamma}{\partial t}-\left(\frac{1}{\rho^{2}} \frac{\partial}{\partial \rho} \rho u\right) \frac{\partial(\rho \gamma)}{\partial \rho}\right.} \\
& \left.-\frac{\partial u}{\partial z} \frac{\partial \gamma}{\partial z}-\sigma \eta_{0} \frac{\partial u}{\partial t} \gamma\right] d g d t \\
+\int_{\Phi^{T}} & {\left[\left(\frac{1}{\rho} \frac{\partial}{\partial \rho} \rho u\right) \gamma \cos (\vec{n}, \vec{\rho})+\frac{\partial u}{\partial z} \gamma \cos (\vec{n}, \vec{z})\right] d s d t=0 . }
\end{aligned}
$$

Let us introduce an arbitrary $\tau \in(0, T)$ and consider the following function:

$$
\gamma(g, t)= \begin{cases}\int_{t}^{\tau} u(g, \zeta) d \zeta ; & 0<t<\tau \\ 0 ; & \tau<t<T\end{cases}
$$

It can be easily verified that $\gamma(g, t)$ has (in $\Omega_{\text {int }}^{T}$ ) the generalized derivatives [44]

$$
\begin{aligned}
& \frac{\partial \gamma(g, t)}{\partial t}= \begin{cases}-u(g, t) ; & 0<t<\tau \\
0 ; & \tau<t<T,\end{cases} \\
& \frac{\partial \gamma(g, t)}{\partial \rho}= \begin{cases}\int_{t}^{\tau} \frac{\partial u(g, \zeta)}{\partial \rho} d \zeta ; & 0<t<\tau \\
0 ; & \tau<t<T,\end{cases} \\
& \frac{\partial \gamma(g, t)}{\partial z}= \begin{cases}\int_{t}^{\tau} \frac{\partial u(g, \zeta)}{\partial z} d \zeta ; & 0<t<\tau \\
0 ; & \tau<t<T .\end{cases}
\end{aligned}
$$

At the same time, we have $\left.\gamma(g, t)\right|_{t=T}=0$. Substituting the function $\gamma(g, t)$ into identity (51), we obtain

$$
\begin{aligned}
\int_{\Omega_{\mathrm{int}}^{\tau}}\left[\varepsilon \frac{\partial u}{\partial t} u+\frac{1}{\rho^{2}}\left(\frac{\partial}{\partial \rho} \rho u\right)\left(\int_{t}^{\tau} \frac{\partial}{\partial \rho} \rho u(\zeta) d \zeta\right)\right. \\
\left.+\frac{\partial u}{\partial z}\left(\int_{t}^{\tau} \frac{\partial}{\partial z} u(\zeta) d \zeta\right)+\sigma \eta_{0} \frac{\partial u}{\partial t} \gamma\right] d g d t \\
-\int_{\Phi^{\tau}}\left[\left(\frac{1}{\rho} \frac{\partial}{\partial \rho} \rho u\right) \gamma \cos (\vec{n}, \vec{\rho})+\frac{\partial u}{\partial z} \gamma \cos (\vec{n}, \vec{z})\right] d s d t=0 .
\end{aligned}
$$

Since [44]

$$
\begin{array}{r}
\int_{\Omega_{\mathrm{int}}^{\tau}}\left[k(g) f(g, t) \int_{t}^{\tau} f(g, \zeta) d \zeta\right] d g d t \\
=\frac{1}{2} \int_{\Omega_{\mathrm{int}}} k(g)\left(\int_{0}^{\tau} f(g, t) d t\right)^{2} d g,
\end{array}
$$

then

$$
\begin{gathered}
\int_{\Omega_{\mathrm{int}}^{\tau}}\left[\frac{1}{\rho^{2}}\left(\frac{\partial}{\partial \rho} \rho u\right)\left(\int_{t}^{\tau} \frac{\partial}{\partial \rho} \rho u(\zeta) d \zeta\right)\right] d g d t \\
=\frac{1}{2} \int_{\Omega_{\mathrm{int}}} \frac{1}{\rho^{2}}\left(\int_{0}^{\tau} \frac{\partial}{\partial \rho} \rho u d t\right)^{2} d g \geq 0, \\
\int_{\Omega_{\mathrm{int}}^{\tau}}\left[\frac{\partial u}{\partial z}\left(\int_{t}^{\tau} \frac{\partial}{\partial z} u(\zeta) d \zeta\right)\right] d g d t \\
=\frac{1}{2} \int_{\Omega_{\mathrm{int}}}\left(\int_{0}^{\tau} \frac{\partial}{\partial z} u d t\right)^{2} d g \geq 0 .
\end{gathered}
$$

By performing partial integration and taking into consideration that $\left.\gamma(g, t)\right|_{t=\tau}=0$ and $\left.u(g, t)\right|_{t=0}=0$, we also obtain

$$
\begin{array}{r}
\int_{\Omega_{\mathrm{int}}^{\tau}}\left[\varepsilon \frac{\partial u}{\partial t} u\right] d g d t=\frac{1}{2} \int_{\Omega_{\mathrm{int}}} \varepsilon[u(g, \tau)]^{2} d g \geq 0, \\
\int_{\Omega_{\mathrm{int}}^{\tau}}\left[\sigma \frac{\partial u}{\partial t} \gamma\right] d g d t=-\int_{\Omega_{\mathrm{int}}^{\tau}}\left[\sigma u \frac{\partial \gamma}{\partial t}\right] d g d t \\
=\int_{\Omega_{\mathrm{int}}^{\tau}} \sigma u^{2} d g d t \geq 0 .
\end{array}
$$


Thus, all the volume integrals entering identity (54) are nonnegative. Show that the integral

$$
\begin{array}{r}
I_{1}(\tau)=-\int_{\Phi^{\tau}}\left[\left(\frac{1}{\rho} \frac{\partial}{\partial \rho} \rho u\right) \gamma \cos (\vec{n}, \vec{\rho})\right. \\
\left.+\frac{\partial u}{\partial z} \gamma \cos (\vec{n}, \vec{z})\right] d s d t
\end{array}
$$

is nonnegative as well. To this end, let us estimate the integral $I_{1}(\tau)$ for the case of $\mathrm{TE}_{0}$-waves, when (see $[3,8]$ )

$$
\begin{array}{cc}
u(g, t)=E_{\phi}, & E_{\rho}=E_{z}=H_{\phi} \equiv 0, \\
\frac{\partial H_{\rho}}{\partial t}=\eta_{0}^{-1} \frac{\partial u}{\partial z}, & \frac{\partial H_{z}}{\partial t}=-\eta_{0}^{-1} \frac{1}{\rho} \frac{\partial(\rho u)}{\partial \rho}
\end{array}
$$

(the case of $\mathrm{TM}_{0}$-waves can be considered similarly). Thus,

$$
\begin{aligned}
I_{1}(\tau) & =\eta_{0} \int_{\Phi^{\tau}}\left[\frac{\partial H_{z}}{\partial t} \gamma \cos (\vec{n}, \vec{\rho})-\frac{\partial H_{\rho}}{\partial t} \gamma \cos (\vec{n}, \vec{z})\right] d s d t \\
& =-\eta_{0} \int_{\Phi^{\tau}}\left[H_{z} \frac{\partial \gamma}{\partial t} \cos (\vec{n}, \vec{\rho})-H_{\rho} \frac{\partial \gamma}{\partial t} \cos (\vec{n}, \vec{z})\right] d s d t \\
& =\eta_{0} \int_{\Phi^{\tau}}\left[H_{z} u \cos (\vec{n}, \vec{\rho})-H_{\rho} u \cos (\vec{n}, \vec{z})\right] d s d t \\
& =\eta_{0} \int_{\Phi^{\tau}}\left[H_{z} E_{\phi} \cos (\vec{n}, \vec{\rho})-H_{\rho} E_{\phi} \cos (\vec{n}, \vec{z})\right] d s d t \\
& =\eta_{0} \int_{\Gamma_{\text {add }} \times(0, \tau)}\left[H_{z} E_{\phi} \cos (\vec{n}, \vec{\rho})-H_{\rho} E_{\phi} \cos (\vec{n}, \vec{z})\right] d s d t \\
& =\eta_{0} \int_{\Gamma_{\text {add }} \times(0, \tau)}([\vec{E} \times \vec{H}] \cdot \vec{n}) d s d t=\eta_{0} I_{2}(\tau) \geq 0 .
\end{aligned}
$$

The last step in the chain of transformations (61) requires explanation. The integral $I_{2}(\tau)$, accurate within a fixed factor, coincides with the electromagnetic field energy radiated from the region $\Omega_{\text {int }} \times[0 \leq \phi \leq 2 \pi]$ during the time $0<t<\tau$ [45]. According to the condition $\left.D_{\text {add }}[u(g, t)]\right|_{g \in \Gamma_{\text {add }}}=0$ (the operator $D_{\text {add }}[\cdots]$, here, is given by (29)-(31) and (40a)(42b)), the functions $\vec{E}=\left\{E_{\rho}, E_{\phi}, E_{z}\right\}$ and $\vec{H}=\left\{H_{\rho}, H_{\phi}, H_{z}\right\}$ correspond to the electromagnetic waves outgoing from the domain $\Omega_{\text {int }}$ and the energy of the outgoing waves cannot be negative.

Then, from (54)-(58) and (61), we have

$$
\int_{\Omega_{\mathrm{int}}} \varepsilon[u(g, \tau)]^{2} d g=\eta_{0} \int_{\Omega_{\mathrm{int}}^{\tau}} \sigma u^{2} d g d t=0,
$$

or, in view of arbitrariness of $\tau$,

$$
u(g, t) \equiv 0 ; \quad g \in \Omega_{\text {int }}, 0<t<T .
$$

Thus, the solution to the modified problem (49) exists and it is unique. This result allows one to construct based on (49) stable and convergent finite-difference or finite-element computational schemes for computing approximate values of the field $U(g, t), g \in \Omega, 0 \leq t \leq T<\infty$ (see [3,31, 36, 42]) and proves the following statement.
Statement. Suppose that problem (4) has a unique solution from $W_{2}^{1}\left(\Omega^{T}\right)$. Then, problem (49) is uniquely solvable in the space $W_{2}^{1}\left(\Omega_{\text {int }}^{T}\right)$, and the closed region problem (20), (49) is equivalent to the unbounded region problem (4).

\section{Conclusion}

In this paper, a problem of efficient truncation of the computational domain of finite-difference or finite-element methods is discussed for axially symmetrical open waveguide structures. The original problem describing pulsed wave scattering on a waveguide discontinuity is an initial boundary value problem formulated in an unbounded domain. The exact absorbing conditions have been derived for artificial boundaries enveloping all sources and scatterers in order to truncate the computational domain and replace the original open problem by an equivalent closed one.

It has been proved that the modified (closed) problem is uniquely solvable if the original (open) problem is wellposed. The solutions to these two problems coincide; that is, these problems are equivalent. This result has been obtained by developing the technique previously used in classical works for studying initial boundary value problems with Dirichlet and Neumann boundary conditions (see, e.g., [36, $44]$ ) and for the first time modified for the open problems with EAC in [9]. It can be now used for most problems of the EAC-method. Conceptually, the proof of the equivalency completes the mathematical justification of the corresponding approach, whose computational efficiency has already been confirmed in a series of works $[3,8,22,24-30]$.

In conclusion, it is worth noting once again that, in contrast to the well-known approximate boundary conditions utilized by finite-difference and finite-element methods, the boundary conditions derived in this paper are exact by construction and do not introduce an additional error into any respective algorithm. This advantage is especially valuable in resonant situations, where numerical simulation requires large running time and calculational errors may grow unpredictably if an open problem is replaced by an insufficiently accurate closed problem.

\section{Conflict of Interests}

The authors declare that there is no conflict of interests regarding the publication of this paper.

\section{References}

[1] A. R. Maikov, A. G. Sveshnikov, and S. A. Yakunin, "A difference scheme for the non-stationary maxwell equations in waveguide systems," USSR Computational Mathematics and Mathematical Physics, vol. 26, no. 3, pp. 130-138, 1986.

[2] K. Y. Sirenko and Y. K. Sirenko, "Exact "absorbing" conditions in initial-boundary value problems in the theory of open waveguide resonators," Computational Mathematics and Mathematical Physics, vol. 45, no. 3, pp. 490-506, 2005.

[3] Y. K. Sirenko, S. Strom, and N. P. Yashina, Modeling and Analysis of Transient Processes in Open Resonant Structures. New Methods and Techniques, Springer, New York, NY, USA, 2007. 
[4] K. Y. Sirenko, Y. K. Sirenko, and N. P. Yashina, "Modeling and analysis of transients in periodic gratings. I. Fully absorbing boundaries for 2-D open problems," Journal of the Optical Society of America A, vol. 27, no. 3, pp. 532-543, 2010.

[5] Y. K. Sirenko and S. Strom, Modern Theory of Gratings. Resonant Scattering: Analysis Techniques and Phenomena, Springer, New York, NY, USA, 2010.

[6] K. Sirenko, V. Pazynin, Y. Sirenko, and H. Bagci, "An FFTaccelerated fdtd scheme with exact absorbing conditions for characterizing axially symmetric resonant structures," Progress in Electromagnetics Research, vol. 111, pp. 331-364, 2011.

[7] O. Shafalyuk, Y. Sirenko, and P. Smith, "Simulation and analysis of transient processes in open axially-symmetrical structures: Method of exact absorbing boundary conditions," in Electromagnetic Waves, V. Zhurbenko, Ed., pp. 99-116, InTech, Rijeka, Croatia, 2011.

[8] V. F. Kravchenko, Y. K. Sirenko, and K. Y. Sirenko, Electromagnetic Wave Transformation and Radiation by the Open Resonant Structures. Modelling and Analysis of Transient and Steady-State Processes, Fizmathlit, Moscow, Russia, 2011 (Russian).

[9] O. Shafalyuk, P. Smith, and L. Velychko, "Rigorous substantiation of the method of exact absorbing conditions in timedomain analysis of open electrodynamic structures," Progress in Electromagnetics Research B, vol. 41, pp. 231-249, 2012.

[10] B. Engquist and A. Majda, "Absorbing boundary conditions for the numerical simulation of waves," Mathematics of Computation, vol. 31, no. 139, pp. 629-651, 1977.

[11] G. Mur, "Absorbing boundary conditions for the finitedifference approximation of the time-domain electromagneticfield equations," IEEE Transactions on Electromagnetic Compatibility, vol. 23, no. 4, pp. 377-382, 1981.

[12] P. A. Tirkas, C. A. Balanis, and R. A. Renaut, "Higher order absorbing boundary conditions for FDTD-method," IEEE Transactions on Antennas and Propagation, vol. 40, no. 10, pp. 1215-1222, 1992.

[13] K. K. Mei and J. Fang, "Superabsorption-a method to improve absorbing boundary conditions," IEEE Transactions on Antennas and Propagation, vol. 40, no. 9, pp. 1001-1010, 1992.

[14] J.-P. Berenger, "A perfectly matched layer for the absorption of electromagnetic waves," Journal of Computational Physics, vol. 114, no. 2, pp. 185-200, 1994.

[15] J. P. Berenger, "Three-dimensional perfectly matched layer for the absorption of electromagnetic waves," Journal of Computational Physics, vol. 127, no. 2, pp. 363-379, 1996.

[16] Z. S. Sacks, D. M. Kingsland, R. Lee, and J. F. Lee, "Perfectly matched anisotropic absorber for use as an absorbing boundary condition," IEEE Transactions on Antennas and Propagation, vol. 43, no. 12, pp. 1460-1463, 1995.

[17] F. L. Teixeira and W. C. Chew, "Finite-difference computation of transient electromagnetic waves for cylindrical geometries in complex media," IEEE Transactions on Geoscience and Remote Sensing, vol. 38, no. 4, pp. 1530-1543, 2000.

[18] S. Abarbanel, D. Gottlieb, and J. S. Hesthaven, "Long time behavior of the perfectly matched layer equations in computational electromagnetics," Journal of Scientific Computing, vol. 17, no. 1-4, pp. 405-422, 2002.

[19] E. Bécache, P. G. Petropoulos, and S. D. Gedney, "On the long-time behavior of unsplit perfectly matched layers," IEEE Transactions on Antennas and Propagation, vol. 52, no. 5, pp. 1335-1342, 2004.
[20] A. Perov, Y. Sirenko, and N. Yashina, "Explicit conditions for virtual boundaries in initial boundary value problems in the theory of wave scattering," Journal of Electromagnetic Waves and Applications, vol. 13, no. 10, pp. 1343-1371, 1999.

[21] Y. K. Sirenko, L. G. Velychko, and F. Erden, "Time-domain and frequency-domain methods combined in the study of open resonance structures of complex geometry," Progress in Electromagnetics Research, vol. 44, pp. 57-79, 2004.

[22] K. Y. Sirenko and V. L. Pazynin, "Axially-symmetrical radiators of pulsed and monochromatic-and-waves," Uspehi Sovremennoy Radioelektroniki, no. 4, pp. 52-69, 2006 (Russian).

[23] L. G. Velychko, Y. K. Sirenko, and O. S. Velychko, "Timedomain analysis of open resonators. Analytical grounds," Progress in Electromagnetics Research, vol. 61, pp. 1-26, 2006.

[24] K. Y. Sirenko, "Slot resonances in xially symmetric radiators of pulse-modulated and monochromatic TM0n-modes," Telecommunications and Radio Engineering, vol. 66, no. 1, pp. 9-21, 2007.

[25] K. Y. Sirenko, "Splitting of super-broadband pulses by simple inhomogeneities of circular and coaxial waveguides," Telecommunications and Radio Engineering, vol. 67, no. 16, pp. 1415$1428,2008$.

[26] I. K. Kuzmitchev, P. M. Melezhyk, V. L. Pazynin et al., "Model synthesis of energy compressors," Radiofizika I Elektronika, vol. 13, no. 2, pp. 166-172, 2008.

[27] L. G. Velychko and Y. K. Sirenko, "Controlled changes in spectra of open quasi-optical resonators," Progress In Electromagnetics Research B, no. 16, pp. 85-105, 2009.

[28] K. Y. Sirenko, Y. K. Sirenko, and N. P. Yashina, "Modeling and analysis of transients in periodic gratings. II. Resonant wave scattering," Journal of the Optical Society of America A, vol. 27, no. 3, pp. 544-552, 2010.

[29] K. Sirenko, V. Pazynin, Y. Sirenko, and H. Bağci, "Compression and radiation of high-power short rf pulses. I. Energy accumulation in direct-flow waveguide compressors," Progress in Electromagnetics Research, vol. 116, pp. 239-270, 2011.

[30] K. Sirenko, V. Pazynin, Y. Sirenko, and H. Bağci, “Compression and radiation of high-power short rf pulses. II. A novel antenna array design with combined compressor/radiator elements," Progress in Electromagnetics Research, vol. 116, pp. 271-296, 2011.

[31] A. Taflove and S. C. Hagness, Computational Electrodynamics: The Finite-Difference Time-Domain Method, Artech House, Boston, Mass, USA, 2000.

[32] Rao and S. M. (ed):, Time Domain Electromagnetics, Academic Press, San Diego, Calif, USA, 1999.

[33] K. Y. Sirenko, "Transport operators in the axially-symmetrical problems of the electrodynamics of pulsed waves," Elektromagnitnye Volny I Elektronnye Sistemy, vol. 11, no. 11, pp. 15-26, 2006 (Russian).

[34] V. F. Kravchenko, K. Y. Sirenko, and Y. K. Sirenko, "Transport operators and exact absorbing conditions in the plane problems of the electrodynamics of pulsed waves for compact open resonators with the waveguide feeder line," Elektromagnitnye Volny I Elektronnye Sistemy, vol. 14, no. 1, pp. 4-19, 2009 (Russian).

[35] V. V. Borisov, Electromagnetic Fields of Transient Currents, St. Petersburg University Press, St. Petersburg, Russia, 1996 (Russian).

[36] O. A. Ladyzhenskaya, The Boundary Value Problems of Mathematical Physics, Springer, New York, NY, USA, 1985.

[37] G. A. Korn and T. M. Korn, Mathematical Handbook for Scientists and Engineers, McGraw-Hill, New York, NY, USA, 1961. 
[38] V. S. Vladimirov, Equations of Mathematical Physics, Dekker, New York, NY, USA, 1971.

[39] M. Abramowitz and I. A. Stegun, Handbook of Mathematical Functions, Dover, New York, NY, USA, 1972.

[40] H. Bateman and A. Erdelyi, Tables of Integral Transforms, vol. 1, McGraw-Hill, New York, NY, USA, 1954.

[41] B. R. Waynberg, Asymptotic Methods in the Equations of Mathematical Physics, Moscow State University Press, Moscow, Russia, 1982 (Russian).

[42] Y. K. Sirenko, V. P. Shestopalov, and N. P. Yashina, "Free oscillations in coaxial-waveguide resonator," Soviet Journal of Communications Technology and Electronics, vol. 32, no. 7, pp. 60-67, 1987.

[43] I. S. Gradshteyn and I. M. Ryzhik, Table of Integrals, Series, and Products, Academic Press, San Diego, Calif, USA, 2000.

[44] V. P. Mikhailov, Partial Differential Equations, Mir Publishers, Moscow, Russia, 1978.

[45] J. D. Jackson, Classical Electrodynamics, John Wiley \& Sons, New York, NY, USA, 1975. 

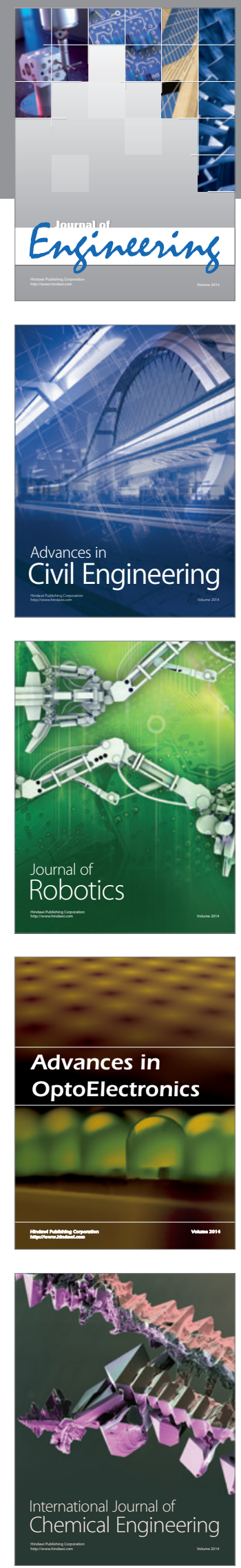

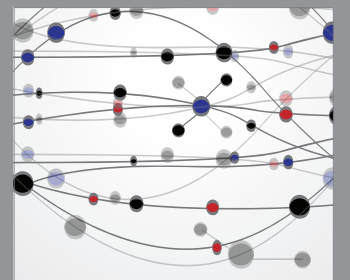

The Scientific World Journal
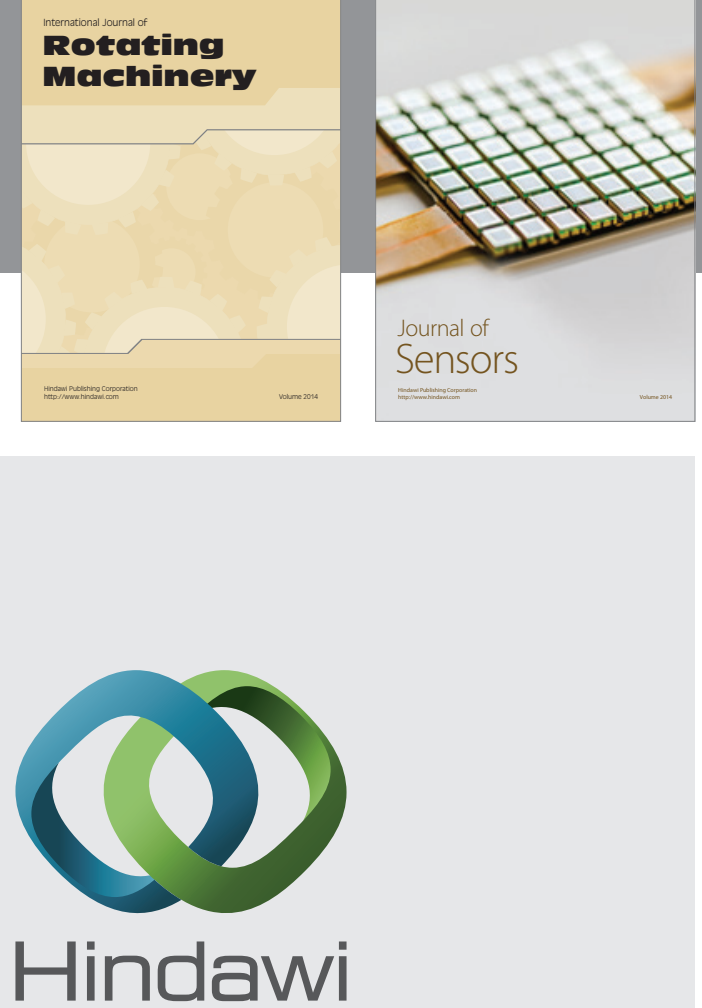

Submit your manuscripts at http://www.hindawi.com
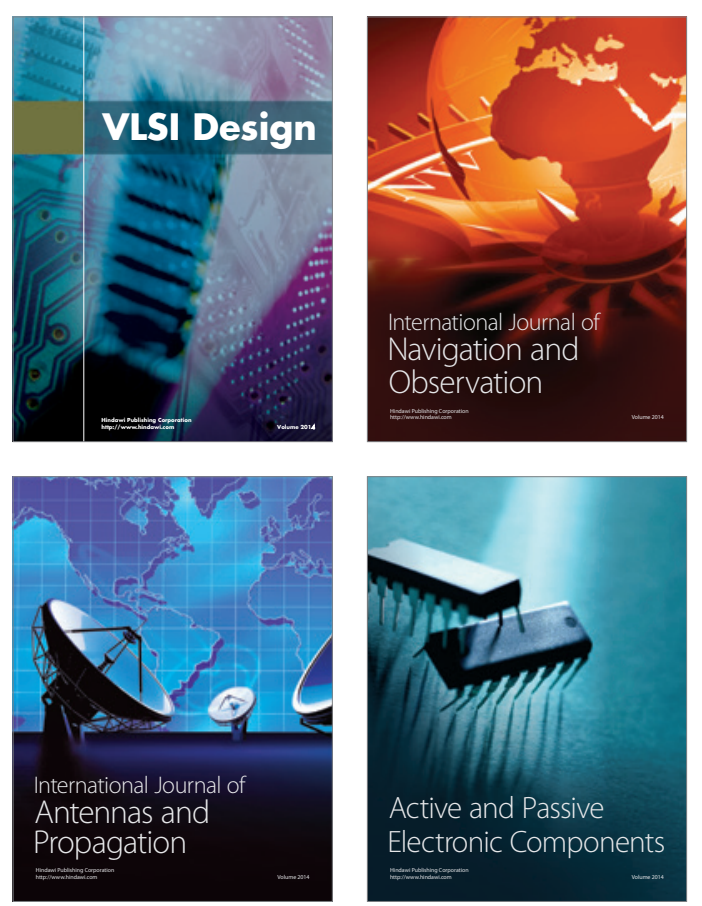
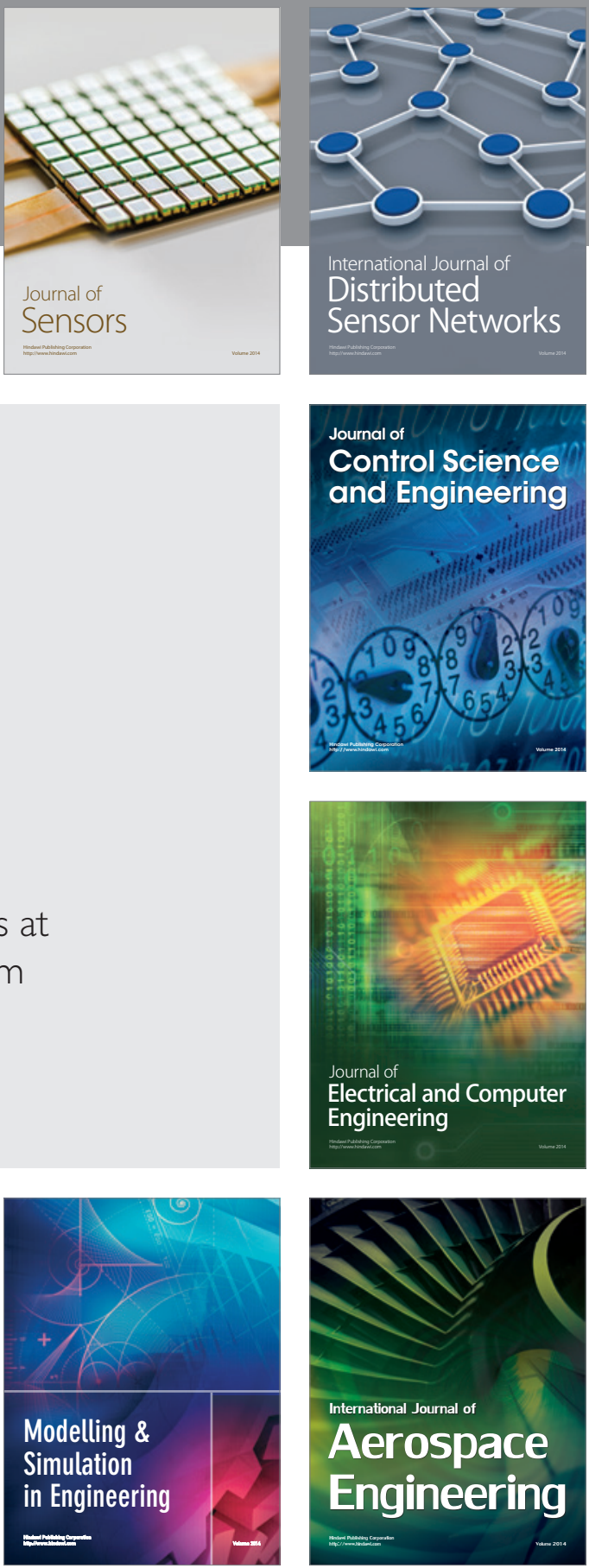

Journal of

Control Science

and Engineering
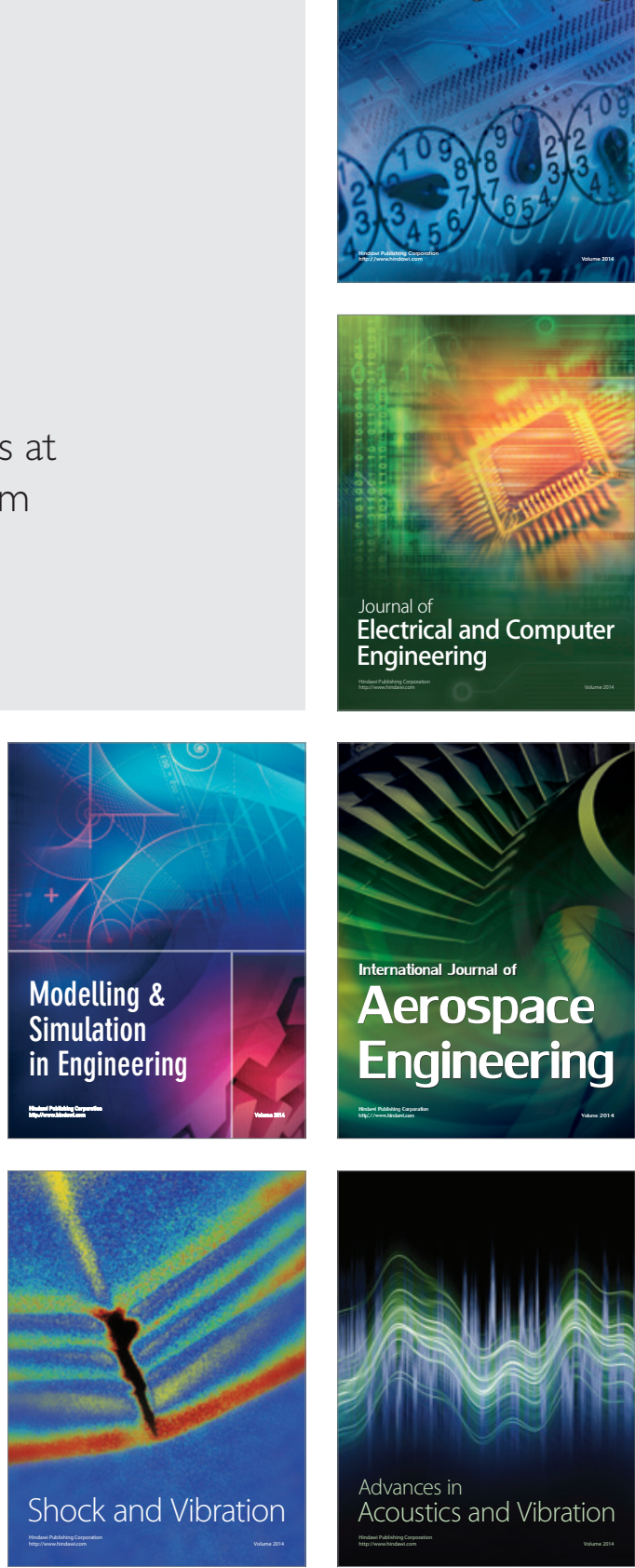